\title{
The health of women and children - an integral part of practice of family and community medicine
}

\author{
$\mathrm{Li} \mathrm{Li}$
}

The practice of family and community medicine covers all spectrum of health, including that of women and children. In this issue of FMCH, volume 6, issue number 3 , four original research articles address various aspects of health in these two subpopulations, highlighting the comprehensiveness of primary healthcare.

Specifically, Siuw et al. [1] examined intraocular pressure [IOP] among premenopausal and postmenopausal women in Malaysia and reported a significant impact on IOP by menopause; Ishola et al. [2] developed and validated a new tool to screen postpartum depression among mothers of preterm babies, which demonstrated meritorious validity in the obstetric population; Ghose and Yaya [3] examined factors influencing self-management behavior among hypertensive women in Ghana and found that socioeconomic status significantly impacts women's adherence to blood pressure-controlling behavior; and Shrivastava and Shrivastava [4] assessed the out-of-pocket expenditure on health care of children younger than 5 years in rural India and found that a large part of out-of-pocket expenditure was for injury and trauma in this age group.

Campbell et al. [5] shared their experience in the development and implementation of an integrated primary care-behavioral health program. Their experience is particularly relevant to such practice in rural areas. The Commentary by Michael D. Fetters reflects on experience of teaching practices in undergraduate medical education in the USA and provides a comprehensive and synthesized perspective and useful reference for other countries in their efforts to develop a family medicine workforce [6].

Finally, the China Focus article by Luo et al. [7] provides a comprehensive assessment of the current workforce of general practitioners [GPs] in China and predicts the number of GPs needed in China moving forward.

I hope our international readership will find these articles informative and useful.

\section{References}

1. Siuw CP, Vasudevan S, Mustapha M. Factors influencing IOP changes in postmenopausal women. Fam Med Community Health 2018;6(3):97-103.

2. Ishola AA, Obasi CC, Sholuke IT. Development and validation of the mothers of preterm babies postpartum depression scale. Fam Med Community Health 2018;6(3):104-14.

3. Ghose B, Yaya S. Blood pressure-controlling behavior in relation to educational level and economic status among hypertensive women in Ghana. Fam Med Community Health 2018;6(3):115-23.

4. Shrivastava SR, Shrivastava PS. A cross-sectional study to assess the out-of-pocket expenditure of families on the health care of children younger than 5 years in a rural area. Fam Med Community Health 2018;6(3):124-30.
CORRESPONDING AUTHOR:

Li Li [李浬], MD, PhD

Department of Family Medicine and Community Health, Case Western Reserve University, 11000 Cedar Avenue, Suite 402, Cleveland, OH 44106-7136, USA

E-mail: 1x162@case.edu

Received 8 August 2018; Accepted 13 August 2018 
5. Campbell K, McKnight L, Vasquez AR. Integrated primary carebehavioral health program development and implementation in a rural context. Fam Med Community Health 2018;6(3):131-41.

6. Fetters MD, Rew J, Heidelbaugh JJ. The role of the teaching practice in undergraduate medical education: a perspective from the United States of America. Fam Med Community Health 2018;6(3):142-53.

7. Wu B, Gong H, Luo Z. Number, distribution, and predicted needed number of general practitioners in China. Fam Med Community Health 2018;6(3):154-9. 